\title{
Chiral magnetic effect in the presence of an external axial-vector field
}

\author{
Maxim Dvornikov* \\ Pushkov Institute of Terrestrial Magnetism, Ionosphere and Radiowave Propagation (IZMIRAN), \\ 108840 Moscow, Troitsk, Russia and Physics Faculty, National Research Tomsk State University, \\ 36 Lenin Avenue, 634050 Tomsk, Russia
}

(Received 5 April 2018; published 21 August 2018)

\begin{abstract}
We study the excitation of the electric current of chiral fermions along the external magnetic field, known as the chiral magnetic effect, in the presence of the background axial-vector field. The calculation of the current is based on the exact solution of the Dirac equation for these fermions accounting for the external fields. First, this solution was obtained for massive particles and, then, we consider the chiral limit, which is used in the anomalous current computation. We obtain that, in this situation, the anomalous current does not contain the direct contribution of the axial-vector field. This result is compared with findings of other authors.
\end{abstract}

DOI: $10.1103 /$ PhysRevD.98.036016

\section{INTRODUCTION}

The evolution of chiral charged particles in external fields reveals multiple quantum phenomena. First, we mention the Adler-Bell-Jackiw anomaly [1], which consists in the nonconservation of the axial current in the presence of an external electromagnetic field. This anomaly was shown in Ref. [2] to be closely related to the excitation of the electric current of massless fermions $\mathbf{J}_{\mathrm{CME}}=\alpha_{\mathrm{em}}\left(\mu_{\mathrm{R}}-\right.$ $\left.\mu_{\mathrm{L}}\right) \mathbf{B} / \pi$ along the external magnetic field $\mathbf{B}$. Here $\alpha_{\mathrm{em}} \approx$ $1 / 137$ is the fine structure constant and $\mu_{\mathrm{R}, \mathrm{L}}$ are the chemical potentials of right and left chiral fermions. This phenomenon was named the chiral magnetic effect (CME) later in Ref. [3]. One can also mention the chiral vortical effect (CVE), described in Ref. [4], which is the generation of the anomalous current in a rotating matter. There are active searches for manifestations of the CME in astrophysics and cosmology [5], as well as in accelerator physics [6].

There is an open question on the influence of the external axial-vector field $V_{5}^{\mu}$ on the magnitude of the anomalous current in the CME. If a homogeneous and isotropic $V_{5}^{\mu}$ is present, the Lagrangian of the interaction of the fermion field $\psi$ with $V_{5}^{\mu}$ can be represented as $\mathcal{L}_{5} \sim \bar{\psi} \gamma \gamma^{\mu} \gamma^{5} \psi V_{5 \mu} \rightarrow$ $\psi^{\dagger} \gamma^{5} \psi V_{5}^{0}$, which shows that the chiral imbalance $\mu_{5}=$ $\left(\mu_{\mathrm{R}}-\mu_{\mathrm{L}}\right) / 2$ could be shifted by $V_{5}^{0}$. Here $\gamma^{\mu}=\left(\gamma^{0}, \gamma\right)$ and $\gamma^{5}=\mathrm{i} \gamma^{0} \gamma^{1} \gamma^{2} \gamma^{3}$ are the Dirac matrices. Thus the CME could

\footnotetext{
maxdvo@izmiran.ru
}

Published by the American Physical Society under the terms of the Creative Commons Attribution 4.0 International license. Further distribution of this work must maintain attribution to the author(s) and the published article's title, journal citation, and DOI. Funded by SCOAP. contain the contribution of $V_{5}^{0} \equiv V_{5}$. This idea was recently implemented in Ref. [7]. However the regularization used in Ref. [7] to compute the divergent integrals was ambiguous. Another possibility for $V_{5}^{\mu}$ to affect the CME is the consideration of the polarization operator of a photon in the fermion plasma under the influence of $V_{5}^{\mu}$. In this case, the polarization operator could acquire the additional term $\Pi_{\mu \nu} \sim \mathrm{i} \varepsilon_{\mu \nu \lambda \rho} V_{5}^{\lambda} k^{\rho}$, where $k^{\rho}$ is the photon momentum and $\varepsilon_{\mu \nu \lambda \rho}$ is the antisymmetric tensor. The appearance of this term is equivalent to the excitation of the current $\mathbf{J} \sim V_{5} \mathbf{B}$, which would be a direct contribution of $V_{5}^{\mu}$ to the CME.

The calculation of the antisymmetric contribution to $\Pi_{\mu \nu}$ in the QED plasma of chiral fermions was made in Ref. [8], where the CME was reproduced. Then, analogous calculations in the presence of an external axial-vector field, e.g., the electroweak interaction with background matter, were carried out in Refs. [9,10]. The calculations in Refs. [9,10] demonstrate that one has the nonzero contribution to the polarization tensor $\Pi_{i j} \sim \mathrm{i} \varepsilon_{i j n} k^{n} V_{5}$, showing that the CME can be influenced by the external axial-vector field $V_{5}^{\mu}$. However, a model with $\mathcal{L}_{5} \neq 0$ is nonrenormalizable and the result of the calculation of $\Pi_{\mu \nu}$ was shown in Ref. [11] to depend on the regularization scheme applied.

Using the perturbative loop expansion, the CVE was found in Ref. [12] to receive no radiative corrections from Yukawa-type interactions. This result can be extended to the CME. However, the arguments against the corrections to the CVE are not applicable in the case of dynamical gauge fields, which can be present in a realistic system. We can also mention that lattice calculations, performed in Ref. [13], show that the CME can get a contribution from an interfermion interaction.

The aim of this work is to find out whether there is an influence of the axial-vector field on the CME. We consider 
a particular example of this axial-vector field in the form of the electroweak interaction with background matter. We start in Sec. II with the description of the motivation for this study. Then, in Sec. III, we calculate the anomalous current along the external magnetic field basing on the exact solution of the Dirac equation in the external fields. Our results are discussed in Sec. IV.

\section{MOTIVATION}

In this section, we compare the results of different methods for the calculation of $\mathbf{J}_{\mathrm{CME}} \| \mathbf{B}$ in the presence of $V_{5}^{\mu}$, which is taken in the form of the electroweak interaction with background matter.

The method of the relativistic quantum mechanics was used for the first time to describe the CME in Ref. [14]. The idea of this method is the following. First, one obtains the exact solution of the Dirac equation for a massless particle in an external magnetic field. Then the electric current is computed as

$$
\mathbf{J}_{\chi}=q\left\langle\bar{\psi}_{\chi} \gamma \psi_{\chi}\right\rangle,
$$

where $\psi_{\chi}$ is the wave function, obtained in the solution of the Dirac equation and $q$ is the particle charge. The averaging $\langle\ldots\rangle$ in Eq. (2.1) is made over the statistical ensemble. The contribution of any chirality $\chi=\mathrm{L}, \mathrm{R}$ is accounted for in Eq. (2.1). This method allows one to take into account the contribution of the external field nonperturbatively. Nevertheless, it is restricted to the constant and homogeneous magnetic field.

The application of the relativistic quantum mechanics method for the study of the CME in the presence of the electroweak parity violating interaction was made in Refs. [15,16]. Let us consider a massless electron, electroweakly interacting with nonmoving and unpolarized background matter under the influence of an external magnetic field along the $z$ axis, $\mathbf{B}=B \mathbf{e}_{z}$. The Lagrangian for such an electron, described by the bispinor $\psi_{e}$, has the form

$$
\mathcal{L}=\bar{\psi}_{e}\left[\gamma_{\mu}\left(\mathrm{i} \partial^{\mu}+e A^{\mu}\right)-\gamma_{0}\left(V_{\mathrm{L}} P_{\mathrm{L}}+V_{\mathrm{R}} P_{\mathrm{R}}\right)\right] \psi_{e},
$$

where $A^{\mu}=(0,0, B x, 0)$ is the vector potential corresponding to the constant and homogeneous magnetic field, $e>0$ is the elementary charge, $P_{\mathrm{L}, \mathrm{R}}=\left(1 \mp \gamma^{5}\right) / 2$ are the chiral projectors, and $V_{\mathrm{L}, \mathrm{R}}$ are the effective potentials of the electroweak interaction of the electron chiral projections with background matter. The explicit form of $V_{\mathrm{L}, \mathrm{R}}$ is given in Ref. [15] for the case of background matter consisting of neutrons and protons.

The energy spectra of the chiral projections of the electron were found in Refs. $[15,16]$ as

$$
E_{\mathrm{L}, \mathrm{R}}=V_{\mathrm{L}, \mathrm{R}}+\mathcal{E}_{0}, \quad \mathcal{E}_{0}=\sqrt{p_{z}^{2}+2 e B n},
$$

where $p_{z}$ is the longitudinal momentum along the magnetic field, and $n=0,1, \ldots$ is the discrete quantum number. If $n=0$ in Eq. (2.3), we have obtained in Refs. $[15,16]$ for left electrons,

$$
E_{\mathrm{L}}^{(n=0)}=V_{\mathrm{L}}+p_{z}, \quad 0<p_{z}<+\infty,
$$

and for right particles,

$$
E_{\mathrm{R}}^{(n=0)}=V_{\mathrm{R}}-p_{z}, \quad-\infty<p_{z}<0 .
$$

In Eqs. (2.4) and (2.5) we assumed, in analogy with the situation when $V_{\mathrm{L}, \mathrm{R}}=0$, that left and right electrons move in a certain direction along the magnetic field.

Using the spectra in Eqs. (2.3)-(2.5) and the wave functions, found in Refs. [15,16], as well as applying the general expression for the current in Eq. (2.1), one finds that only the lowest energy level with $n=0$ contributes to the current, giving one its nonzero component along the magnetic field as

$$
\mathbf{J}=\frac{2 \alpha_{\mathrm{em}}}{\pi}\left(\mu_{5}+V_{5}\right) \mathbf{B},
$$

where $\alpha_{\mathrm{em}}=e^{2} / 4 \pi$ is the fine structure constant and $V_{5}=$ $\left(V_{\mathrm{L}}-V_{\mathrm{R}}\right) / 2$ is the contribution of the electroweak interaction.

The result in Eq. (2.6) was criticized in Ref. [17], where it was found that

$$
\mathbf{J}=\frac{2 \alpha_{\mathrm{em}}}{\pi} \mu_{5} \mathbf{B} \equiv \mathbf{J}_{\mathrm{CME}}
$$

even in the presence of the background electroweak matter, i.e., when $V_{\mathrm{L}, \mathrm{R}} \neq 0$. The authors of Ref. [17] used the alternative derivation of the CME based on the energy balance in the motion of a massless charged particle in parallel electric and magnetic fields, previously proposed in Ref. [2]. Note that, even if one uses the method of Ref. [2] to derive the CME in the presence the electroweakly interacting matter and accounts for the dispersion relation in Eqs. (2.4) and (2.5), the expression for the current in Eq. (2.6) can be reproduced [18].

The expression for the current in Eq. (2.7) was also derived in Ref. [19], where the CME in the presence of the axial-vector field was studied using the chiral hydrodynamics approach, which was developed earlier in Ref. [20]. No explicit contribution of the electroweak interaction to the current, like in Eq. (2.6), was found in Ref. [19].

The apparent contradiction between the relativistic quantum mechanics method in Refs. [15,16] and other approaches [17,19] for the description of the CME in the presence of the axial-vector external field requires a special analysis. 


\section{ANOMALOUS CURRENT IN THE PRESENCE OF THE ELECTROWEAK INTERACTION WITH MATTER}

To analyze the contribution of the parity violating interaction to the CME, using the relativistic quantum mechanics approach, we start with the consideration of massive particles interacting with the axial-vector and magnetic fields and then discuss the chiral limit. As in Sec. II, we consider the electroweak interaction of an electron with background matter. Since the relativistic quantum mechanics method deals with an exact solution of the Dirac equation in an external field, the corresponding solution should be utilized. For the first time the Dirac equation for a massive electron, electroweakly interacting with background matter under the influence of an external magnetic field, was solved in Ref. [21]. Then this solution was used in Ref. [22] to compute the induced current along the magnetic field.

Thus, instead of the Lagrangian in Eq. (2.2), we discuss the following Lagrangian:

$\mathcal{L}=\bar{\psi}_{e}\left[\gamma_{\mu}\left(\mathrm{i} \partial^{\mu}+e A^{\mu}\right)-m-\gamma_{0}\left(V_{\mathrm{L}} P_{\mathrm{L}}+V_{\mathrm{R}} P_{\mathrm{R}}\right)\right] \psi_{e}$,

where $m$ is the electron mass. The remaining parameters have the same meaning as in Sec. II.

Let us look for the solution of the Dirac equation, which results from Eq. (3.1), in the form

$$
\psi_{e}=\exp \left(-\mathrm{i} E t+\mathrm{i} p_{y} y+\mathrm{i} p_{z} z\right) \psi_{x}
$$

where $\psi_{x}=\psi(x)$ is the bispinor which depends on $x$ and $p_{y, z}$ are the momentum projections along the $y$ and $z$ axes. We choose the chiral representation of the Dirac matrices [23],

$\gamma^{\mu}=\left(\begin{array}{cc}0 & -\sigma^{\mu} \\ -\bar{\sigma}^{\mu} & 0\end{array}\right), \quad \sigma^{\mu}=\left(\sigma_{0},-\sigma\right)$,

$\bar{\sigma}^{\mu}=\left(\sigma_{0}, \boldsymbol{\sigma}\right)$,

where $\sigma_{0}$ is the unit $2 \times 2$ matrix and $\sigma$ are the Pauli matrices. Using Eq. (3.3), we can represent $\psi_{x}$ in the form [22]

$$
\psi_{x}^{\mathrm{T}}=\left(C_{1} u_{n-1}, \mathrm{i} C_{2} u_{n}, C_{3} u_{n-1}, \mathrm{i} C_{4} u_{n}\right),
$$

where $C_{i}, i=1, \ldots, 4$, are the spin coefficients,

$u_{n}(\eta)=\left(\frac{e B}{\pi}\right)^{1 / 4} \exp \left(-\frac{\eta^{2}}{2}\right) \frac{H_{n}(\eta)}{\sqrt{2^{n} n !}}, \quad n=0,1, \ldots$,

are the Hermite functions, $H_{n}(\eta)$ are the Hermite polynomials, and $\eta=\sqrt{e B} x+p_{y} / \sqrt{e B}$.
The energy spectrum for $n>0$ reads [21,22]

$$
E=\bar{V}+\lambda \mathcal{E}, \quad \mathcal{E}=\sqrt{\left(\mathcal{E}_{0}+s V_{5}\right)^{2}+m^{2}},
$$

where $s= \pm 1$ is the discrete quantum number dealing with the spin operator [21], $\mathcal{E}_{0}$ is defined in Eq. (2.3), $\bar{V}=$ $\left(V_{\mathrm{L}}+V_{\mathrm{R}}\right) / 2$, and $\lambda= \pm 1$ is the sign of the energy; i.e., the electron energy reads $E_{e}=E(\lambda=+1)=\mathcal{E}+\bar{V}$, and the positron energy has the form $E_{\bar{e}}=-E(\lambda=-1)=\mathcal{E}-\bar{V}$. For $n=0$, one has [22]

$$
E=\bar{V}+\lambda \mathcal{E}, \quad \mathcal{E}=\sqrt{\left(p_{z}+V_{5}\right)^{2}+m^{2}} .
$$

Note that, at $n=0$, there is only one spin state of the electron.

The spin coefficients obey the system [22],

$$
\begin{aligned}
& \left(\mathcal{E} \mp p_{z} \pm V_{5}\right) C_{1,3} \mp \sqrt{2 e B n} C_{2,4}+m C_{3,1}=0, \\
& \left(\mathcal{E} \pm p_{z} \pm V_{5}\right) C_{2,4} \mp \sqrt{2 e B n} C_{1,3}+m C_{4,2}=0,
\end{aligned}
$$

where we consider the particle (electron) degrees of freedom, $\lambda=1$. Since we are mainly interested in the dynamics of electrons at the lowest energy level, we should set $n=0$ in Eq. (3.8). It results from Eq. (3.4) that, in this situation, $C_{1}=C_{3}=0$ to avoid the appearance of Hermite functions with negative indices.

If, besides setting $n=0$ in Eq. (3.8), we approach to the limit $m \rightarrow 0$ there, one gets

$$
\left(\mathcal{E}+p_{z}+V_{5}\right) C_{2}=0, \quad \text { or } \quad\left\{\begin{array}{l}
\mathcal{E}=-p_{z}-V_{5}, \\
C_{2} \neq 0, \text { and } C_{4}=0,
\end{array}\right.
$$

$$
\left(\mathcal{E}-p_{z}-V_{5}\right) C_{4}=0, \quad \text { or } \quad\left\{\begin{array}{l}
\mathcal{E}=p_{z}+V_{5}, \\
C_{4} \neq 0, \text { and } C_{2}=0 .
\end{array}\right.
$$

We can see that Eq. (3.9) corresponds to a right electron and Eq. (3.10) to a left one.

The energy spectrum in Eq. (3.7) in the limit $m \rightarrow 0$ reads

$$
\mathcal{E}=\left|p_{z}+V_{5}\right| \text {. }
$$

Comparing Eq. (3.11) with Eqs. (3.9) and (3.10), we obtain that for a right electron

$$
\left|p_{z}+V_{5}\right|=-p_{z}-V_{5}, \quad \text { or } \quad p_{z}<-V_{5},
$$

and 


$$
\left|p_{z}+V_{5}\right|=p_{z}+V_{5}, \quad \text { or } \quad p_{z}>-V_{5},
$$

for a left particle.

Therefore the total energy of a left electron at the lowest energy level has the form

$$
E_{e \mathrm{~L}}^{(n=0)}=V_{\mathrm{L}}+p_{z}, \quad-V_{5}<p_{z}<+\infty,
$$

and

$$
E_{e \mathrm{R}}^{(n=0)}=V_{\mathrm{R}}-p_{z}, \quad-\infty<p_{z}<-V_{5},
$$

of a right particle. Comparing Eqs. (3.14) and (3.15) with Eqs. (2.4) and (2.5), we can see that the form of the spectrum at $n=0$, obtained here, formally coincides with that used in Refs. $[15,16]$. However, the range of the $p_{z}$ variation is different.

To complete the solution of the Dirac equation at $n=0$ and $m \rightarrow 0$ we should fix the remaining spin coefficients. One gets that

$$
C_{2}^{(\mathrm{R})}=C_{4}^{(\mathrm{L})}=\frac{1}{2 \pi}, \quad C_{2}^{(\mathrm{L})}=C_{4}^{(\mathrm{R})}=0,
$$

which results from the normalization condition

$\int \mathrm{d}^{3} x \psi_{p_{y} p_{z} n}^{\dagger} \psi_{p_{y}^{\prime} p_{z}^{\prime} n^{\prime}}=\delta\left(p_{y}-p_{y}^{\prime}\right) \delta\left(p_{z}-p_{z}^{\prime}\right) \delta_{n n^{\prime}}$,

of the total wave function.

The wave function of a positron can be obtained from Eqs. (3.2) and (3.4) by applying the charge conjugation $\psi_{\bar{e}}=\mathrm{i} \gamma^{2} \psi_{e}^{*}$ and setting $\lambda=-1$ in Eq. (3.6). Finally one has

$$
\begin{aligned}
\psi_{\bar{e}}^{\mathrm{T}}= & \exp \left(-\mathrm{i} E_{\bar{e}} t-\mathrm{i} p_{y} y-\mathrm{i} p_{z} z\right) \\
& \times\left(-\mathrm{i} C_{4} u_{n},-C_{3} u_{n-1}, \mathrm{i} C_{2} u_{n}, C_{1} u_{n-1}\right),
\end{aligned}
$$

where the coefficients $C_{i}$ obey the system in Eq. (3.8).

If $n=0$, we obtain on the basis of Eqs. (3.18) and (3.7) that

$\psi_{\bar{e} \mathrm{R}}^{(n=0)}=\exp \left(-\mathrm{i} E_{\bar{e} \mathrm{R}} t-\mathrm{i} p_{y} y-\mathrm{i} p_{z} z\right) \times \frac{\mathrm{i} u_{0}}{2 \pi}(-1,0,0,0)^{\mathrm{T}}$,

where

$$
E_{\bar{e} \mathrm{R}}^{(n=0)}=p_{z}-V_{\mathrm{R}}, \quad-V_{5}<p_{z}<+\infty
$$

is the energy of right positrons at the lowest energy level. For left positrons one has

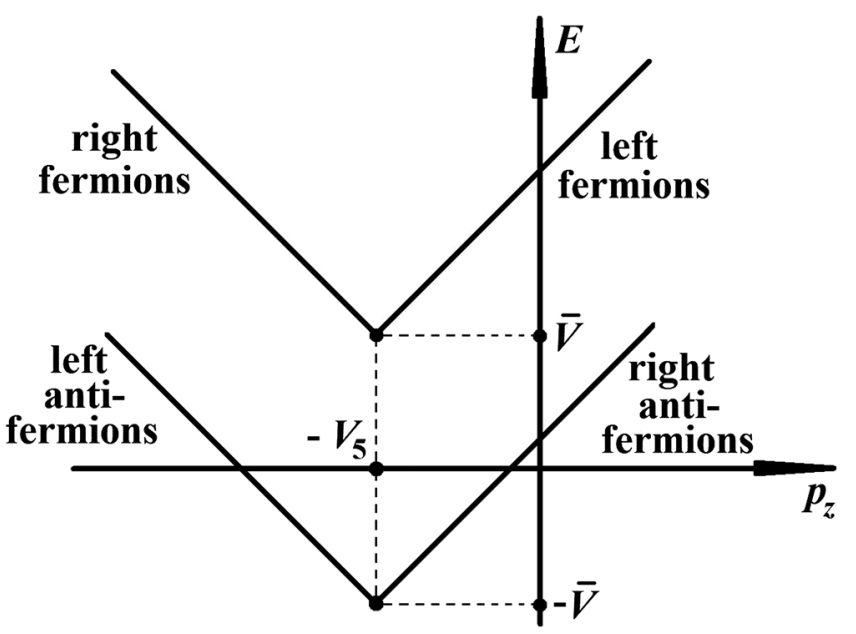

FIG. 1. The energy spectrum of massless left and right electrons/positrons at the lowest energy level with $n=0$ electroweakly interacting with background matter. This plot corresponds to Eqs. (3.14), (3.15), (3.20), and (3.22).

$\psi_{\bar{e} \mathrm{~L}}^{(n=0)}=\exp \left(-\mathrm{i} E_{\bar{e} \mathrm{~L}} t-\mathrm{i} p_{y} y-\mathrm{i} p_{z} z\right) \times \frac{\mathrm{i} u_{0}}{2 \pi}(0,0,1,0)^{\mathrm{T}}$,

where

$$
E_{\bar{e} \mathrm{~L}}^{(n=0)}=-p_{z}-V_{\mathrm{L}}, \quad-\infty<p_{z}<-V_{5}
$$

is the energy of left positrons at the lowest energy level. The positron wave functions in Eqs. (3.19) and (3.21) satisfy the normalization condition in Eq. (3.17).

The energy spectrum for electrons and positrons at the lowest energy level is shown in Fig. 1. One can see that there is no gap between the dispersion relations of left and right electrons/positrons, predicted in Refs. [15,16,18]; i.e., the assumption that $E_{\mathrm{Lmin}}^{(n=0)} \neq E_{\mathrm{Rmin}}^{(n=0)}$ at $p_{z}=0$ [see Eqs. (2.4) and (2.5)] is incorrect. In the presence of the electroweak matter, the spectrum of massless electrons/ positrons with $n=0$ is parallel transported to the point $\left(p_{z}=-V_{5}, E=\bar{V}\right)$ for electrons and to $\left(p_{z}=-V_{5}\right.$, $E=-\bar{V})$ for positrons from the point $\left(p_{z}=0, E=0\right)$ corresponding to the vacuum case.

According to Eq. (2.1), the contributions of left and right electrons at the lowest energy level to the current are

$\mathbf{J}_{e \mathrm{~L}, \mathrm{R}}^{(n=0)}=-e \int \mathrm{d} p_{y} \mathrm{~d} p_{z} \bar{\psi}_{e \mathrm{~L}, \mathrm{R}} \gamma \psi_{e \mathrm{~L}, \mathrm{R}} f\left(E_{e \mathrm{~L}, \mathrm{R}}^{(n=0)}-\mu_{\mathrm{L}, \mathrm{R}}\right)$,

where $f(E)=[\exp (\beta E)+1]^{-1}$ is the Fermi-Dirac distribution function, $\beta=1 / T$ is the reciprocal temperature, and $\mu_{\mathrm{L}, \mathrm{R}}$ are the chemical potentials of left and right particles. First we notice that the components of the current, transverse with respect to $\mathbf{B}$, are vanishing. Performing the 
integration over $-\infty<p_{y}<+\infty$ and accounting for Eqs. (3.14)-(3.16), on the basis of Eq. (3.23) we obtain the expression for the total current of electrons $\mathbf{J}_{e}^{(n=0)}=$ $\mathbf{J}_{\mathrm{L}}^{(n=0)}+\mathbf{J}_{\mathrm{R}}^{(n=0)}$ at $n=0$,

$$
\begin{aligned}
\mathbf{J}_{e}^{(n=0)}= & \frac{e^{2} \mathbf{B}}{(2 \pi)^{2}}\left[\int_{-\infty}^{-V_{5}} \mathrm{~d} p_{z} f\left(-p_{z}+V_{\mathrm{R}}-\mu_{\mathrm{R}}\right)\right. \\
& \left.-\int_{-V_{5}}^{+\infty} \mathrm{d} p_{z} f\left(p_{z}+V_{\mathrm{L}}-\mu_{\mathrm{L}}\right)\right] \\
= & \frac{e^{2} \mathbf{B}}{(2 \pi)^{2}} \int_{0}^{+\infty} \mathrm{d} p\left[f\left(p+\bar{V}-\mu_{\mathrm{R}}\right)\right. \\
& \left.-f\left(p+\bar{V}-\mu_{\mathrm{L}}\right)\right] .
\end{aligned}
$$

Analogously to Refs. [15,16] one can show that higher energy levels with $n>0$ do not contribute to the current. Thus we omit the superscript in Eq. (3.24) for brevity.

The positron contribution to the current $\mathbf{J}_{\bar{e}}$ can be obtained analogously to Eq. (3.23) as

$\mathbf{J}_{\bar{e} \mathrm{~L}, \mathrm{R}}^{(n=0)}=e \int \mathrm{d} p_{y} \mathrm{~d} p_{z} \bar{\psi}_{\bar{e} \mathrm{~L}, \mathrm{R}} \gamma \psi_{\bar{e} \mathrm{~L}, \mathrm{R}} f\left(E_{\bar{e} \mathrm{~L}, \mathrm{R}}^{(n=0)}+\mu_{\mathrm{L}, \mathrm{R}}\right)$.

Using Eqs. (3.19)-(3.22), we derive, on the basis of Eq. (3.25),

$$
\begin{aligned}
\mathbf{J}_{\bar{e}}^{(n=0)}= & \frac{e^{2} \mathbf{B}}{(2 \pi)^{2}}\left[\int_{-\infty}^{-V_{5}} \mathrm{~d} p_{z} f\left(-p_{z}-V_{\mathrm{L}}+\mu_{\mathrm{L}}\right)\right. \\
& \left.-\int_{-V_{5}}^{+\infty} \mathrm{d} p_{z} f\left(p_{z}-V_{\mathrm{R}}+\mu_{\mathrm{R}}\right)\right] \\
= & \frac{e^{2} \mathbf{B}}{(2 \pi)^{2}} \int_{0}^{+\infty} \mathrm{d} p\left[f\left(p-\bar{V}+\mu_{\mathrm{L}}\right)\right. \\
& \left.-f\left(p-\bar{V}+\mu_{\mathrm{R}}\right)\right],
\end{aligned}
$$

for the total contribution to the current from positrons at the lowest energy level.

Using Eqs. (3.24) and (3.26), we obtain that the total current $\mathbf{J}=\mathbf{J}_{e}+\mathbf{J}_{\bar{e}}$ reads

$$
\begin{aligned}
\mathbf{J}= & \frac{e^{2} \mathbf{B}}{(2 \pi)^{2}} \int_{0}^{+\infty} \mathrm{d} p\left[f\left(p+\bar{V}-\mu_{\mathrm{R}}\right)-f\left(p-\bar{V}+\mu_{\mathrm{R}}\right)\right. \\
& \left.-f\left(p+\bar{V}-\mu_{\mathrm{L}}\right)+f\left(p-\bar{V}+\mu_{\mathrm{L}}\right)\right] \\
= & \frac{2 \alpha_{\mathrm{em}}}{\pi} \mu_{5} \mathbf{B},
\end{aligned}
$$

which is in agreement with Eq. (2.7).

\section{DISCUSSION}

In the present work, we have elaborated the improved derivation of the anomalous current of massless charged fermions, interacting with an axial-vector field under the

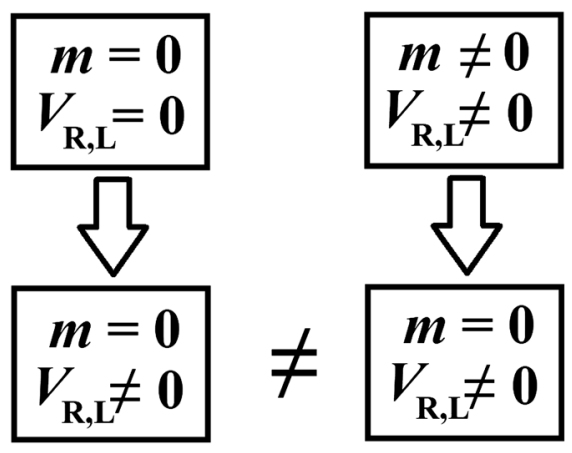

FIG. 2. Schematic diagram showing that the system with $m=0$ and $V_{\mathrm{R}, \mathrm{L}} \neq 0$, which is studied while considering the CME, can be prepared in two nonequivalent ways. One can start with a system of massless particles without the electroweak interaction (upper left box) and then turn on the electroweak interaction (lower left box). This scenario is implemented in Refs. [15,16,18] and gives the current in Eq. (2.6). However, this approach is not equivalent to that, where one, first, starts with massive particles with the electroweak interaction (upper right box) and then "turns off" the particle mass (lower right box). The latter approach results in the current in Eq. (2.7).

influence of the external magnetic field, induced along this magnetic field. We have chosen a particular example of the axial-vector field as the electroweak interaction of an electron with nonmoving and unpolarized background matter. Unlike Refs. [17,19], here we have used the method of the relativistic quantum mechanics, originally proposed in Ref. [14] to describe the CME.

Utilizing the exact solution of the Dirac equation, found in Refs. [21,22], we have shown in Sec. III that the axialvector field does not contribute to the current $\mathbf{J} \| \mathbf{B}$; cf. Eq. (3.27). The value of the current coincides with the prediction of the CME in Eq. (2.7) even in the case when chiral fermions electroweakly interact with background matter, confirming the findings of Refs. [17,19].

To obtain this result in frames of the relativistic quantum mechanics one has to consider the solution of the Dirac equation for a massive electron in the external fields and then approach the limit $m \rightarrow 0$. If one sets $m=0$ in the Dirac equation from the very beginning, i.e., if one considers the chiral Lagrangian in Eq. (2.2), one obtains the current in Eq. (2.6) as in Refs. [15,16], which is inconsistent with the results of Refs. [17,19]. Thus we conclude that the system of chiral fermions, where the external axial-vector field is present, can be prepared in two nonequivalent ways. This fact is reflected in Fig. 2.

It is also interesting to notice that, at $n=0$, particles of a certain chirality, say left-handed, are indirectly affected by the parameters corresponding to the opposite (i.e., righthanded) chirality. It can be seen in Eqs. (3.14) and (3.15). Indeed, if one adiabatically changes $V_{\mathrm{L}}$, not only $E_{\mathrm{L}}^{(n=0)}$ but also $E_{\mathrm{R}}^{(n=0)}$ will be modified since the range of the $p_{z}$ variation in $E_{\mathrm{R}}^{(n=0)}$ depends on $V_{5}$. One would naively 
expect that left- and right-handed electrons behave totally independently for purely massless particles.

The effect of the change of the particle momentum in the presence of the electroweak interaction [see Eq. (3.11)] was known previously. In the case when a particle moves in a background electroweak matter, both particle energy and its momentum acquire the contributions $\sim G_{\mathrm{F}}$, where $G_{\mathrm{F}}$ is the Fermi constant. It results, e.g., in the appearance of the ponderomotive force in the situation of the anisotropic matter with inhomogeneous density [24].

We also mention that one does not need to involve the concept of the Chern-Simons current [25], as suggested in Ref. [17], to reconcile the results for the derivation of the $\mathrm{CME}$ in the presence of the axial-vector field based on the relativistic quantum mechanics [14] and the energy balance arguments [2]. We can obtain the coinciding results just by using the correct energy spectrum of massless electrons at the lowest energy level; cf. Eqs. (3.14) and (3.15).

Since the electroweak interaction of chiral electrons with a homogeneous neutron matter does not contribute to the $\mathrm{CME}$, one cannot expect the instability of the magnetic field and the amplification of the field in a neutron star (NS) to the magnetar strength, predicted in Refs. $[15,16]$. At the absence of $V_{5}$-contribution to the $\mathrm{CME}$, one has the following qualitative behavior of the magnetic field in NS. If the initial chiral imbalance $\mu_{5}$ of ultrarelativistic electrons is present in the system, it is washed out very rapidly because of the helicity flip in electron collisions. The chiral imbalance does not recover since there is no $V_{5}$ driver in the $\mu_{5}$ evolution equation. Since $\mu_{5} \rightarrow 0$, the densities of the magnetic energy and the magnetic helicity are not affected by the CME. Therefore the evolution of the magnetic field does not reveal an instability. Hence the magnetic field in NS can only experience a slow exponential decay because of the finite electric conductivity of NS matter. The direct numerical simulations confirm this magnetic field behavior.

Since the model of the magnetic field generation in magnetars driven by the electroweak interaction of electrons with background nucleons is likely to be invalid, other mechanisms for the explanation of magnetic fields in magnetars, different from that proposed in Refs. [15,16], should be put forward. The classical magnetohydrodynamics (MHD) dynamo is unlikely to be sufficient for the generation of strong magnetic fields in magnetars [26]. Hence one should probably look for the solution of the magnetars problem in frames of the elementary particle physics, e.g., involving chiral phenomena.

In this respect, we can mention Ref. [27], where the CVE is used to generate the fluid helicity of plasma under the influence of strong neutrino fluxes in a nascent NS. Then this fluid helicity can be converted to a helical magnetic field. To solve the problem of the magnetic field scale, it was suggested in Ref. [28] to implement the inverse cascade in chiral MHD in proto-NS matter. The neutrino driven creation of $\mu_{5}$ outside the neutrino-sphere in protoNS was proposed in Ref. [29]. The generation of magnetic fields in a magnetar owing to the CME, based on this $\mu_{5}$, was also studied in Ref. [29]. However, analogously to Ref. [27], that model encounters a problem of the small scale of the magnetic field created.

The system of equations of chiral MHD was formulated in Ref. [30]; the effects of turbulence in such media were also studied there. The direct numerical simulations of the chiral MHD dynamos were performed in Ref. [31]. The implication of the obtained results for the description of the magnetic fields generation in proto-NS was discussed in Refs. [30,31].

A possible explanation of magnetar bursts [26] based on the magnetic field reconnection owing to accounting of quantum terms in the magnetic helicity evolution was recently proposed in Ref. [32]. Note that these quantum corrections to the classical MHD arise from the nonzero mass terms in the Adler-Bell-Jackiw anomaly. It means that one does not need to consider the chiral symmetry restoration in the model in Ref. [32].

\section{ACKNOWLEDGMENTS}

I thank the Tomsk State University Competitiveness Improvement Program and RFBR (Grant No. 18-0200149a) for partial support.
[1] M. E. Peskin and D. V. Schröder, An Introduction to Quantum Field Theory (Reading, Massachusetts, 1995), pp. 659-667.

[2] H. B. Nielsen and M. Ninomiya, The Adler-Bell-Jackiw anomaly and Weyl fermions in a crystal, Phys. Lett. 130B, 389 (1983).

[3] K. Fukushima, D. E. Kharzeev, and H. J. Warringa, The chiral magnetic effect, Phys. Rev. D 78, 074033 (2008).
[4] A. Vilenkin, Macroscopic parity violating effects: Neutrino fluxes from rotating black holes and in rotating thermal radiation, Phys. Rev. D 20, 1807 (1979).

[5] G. Sigl, Astroparticle Physics: Theory and Phenomenology (Atlantis, Paris, 2017).

[6] V. Koch, S. Schlichting, V. Skokov, P. Sorensen, J. Thomas, S. Voloshin, G. Wang, and H.-U. Yee, Status of the chiral magnetic effect and collisions of isobars, Chin. Phys. C 41, 072001 (2017). 
[7] A. F. Bubnov, N. V. Gubina, and V. Ch. Zhukovsky, Vacuum current induced by an axial-vector condensate and electron anomalous magnetic moment in a magnetic field, Phys. Rev. D 96, 016011 (2017).

[8] Y. Akamatsu and N. Yamamoto, Chiral Plasma Instabilities, Phys. Rev. Lett. 111, 052002 (2013).

[9] A. Boyarsky, O. Ruchayskiy, and M. Shaposhnikov, LongRange Magnetic Fields in the Ground State of the Standard Model Plasma, Phys. Rev. Lett. 109, 111602 (2012).

[10] M. Dvornikov and V. B. Semikoz, Instability of magnetic fields in electroweak plasma driven by neutrino asymmetries, J. Cosmol. Astropart. Phys. 05 (2014) 002.

[11] R. Jackiw and V. A. Kostelecký, Radiatively Induced Lorentz and CPT Violation in Electrodynamics, Phys. Rev. Lett. 82, 3572 (1999).

[12] S. Golkar and T. D. Son, (Non)-renormalization of the chiral vortical effect coefficient, J. High Energy Phys. 02 (2015) 169.

[13] P. V. Buividovich, M. Puhr, and S. N. Valgushev, Chiral magnetic conductivity in an interacting lattice model of parity-breaking Weyl semimetal, Phys. Rev. B 92, 205122 (2015).

[14] A. Vilenkin, Equilibrium parity-violating current in a magnetic field, Phys. Rev. D 22, 3080 (1980).

[15] M. Dvornikov and V. B. Semikoz, Magnetic field instability in a neutron star driven by the electroweak electron-nucleon interaction versus the chiral magnetic effect, Phys. Rev. D 91, 061301 (2015).

[16] M. Dvornikov and V.B. Semikoz, Generation of the magnetic helicity in a neutron star driven by the electroweak electron-nucleon interaction, J. Cosmol. Astropart. Phys. 05 (2015) 032.

[17] D. B. Kaplan, S. Reddy, and S. Sen, Energy conservation and the chiral magnetic effect, Phys. Rev. D 96, 016008 (2017).

[18] M. Dvornikov and V. B. Semikoz, Chiral magnetic effect in the presence of electroweak interactions as a quasiclassical phenomenon, Mod. Phys. Lett. A 33, 1850043 (2018).
[19] A. V. Sadofyev and M. V. Isachenkov, The chiral magnetic effect in hydrodynamical approach, Phys. Lett. B 697, 404 (2011).

[20] D. T. Son and P. Surówka, Hydrodynamics with Triangle Anomalies, Phys. Rev. Lett. 103, 191601 (2009).

[21] I. A. Balantsev, Yu. V. Popov, and A. I. Studenikin, On the problem of relativistic particles motion in strong magnetic field and dense matter, J. Phys. A 44, 255301 (2011).

[22] M. Dvornikov, Role of particle masses in the magnetic field generation driven by the parity violating interaction, Phys. Lett. B 760, 406 (2016).

[23] C. Itzykson and J.-B. Zuber, Quantum Field Theory (McGraw-Hill, New York, 1980), pp. 691-696.

[24] L. O. Silva, R. Bingham, J. M. Dawson, J. T. Mendonça, and P. K. Shukla, Neutrino Driven Streaming Instabilities in a Dense Plasma, Phys. Rev. Lett. 83, 2703 (1999).

[25] K. Landsteiner, Notes on anomaly induced transport, Acta Phys. Pol. B 47, 2617 (2016).

[26] S. Mereghetti, J. Pons, and A. Melatos, Magnetars: Properties, origin and evolution, Space Sci. Rev. 191, 315 (2015).

[27] N. Yamamoto, Chiral transport of neutrinos in supernovae: Neutrino-induced fluid helicity and helical plasma instability, Phys. Rev. D 93, 065017 (2016).

[28] N. Yamamoto, Scaling laws in chiral hydrodynamic turbulence, Phys. Rev. D 93, 125016 (2016).

[29] G. Sigl and N. Leite, Chiral magnetic effect in protoneutron stars and magnetic field spectral evolution, J. Cosmol. Astropart. Phys. 01 (2016) 025.

[30] I. Rogachevskii, O. Ruchayskiy, A. Boyarsky, J. Fröhlich, N. Kleeorin, A. Brandenburg, and J. Schober, Laminar and turbulent dynamos in chiral magnetohydrodynamics. I. Theory, Astrophys. J. 846, 153 (2017).

[31] J. Schober, I. Rogachevskii, A. Brandenburg, A. Boyarsky, J. Fröhlich, O. Ruchayskiy, and N. Kleeorin, Laminar and turbulent dynamos in chiral magnetohydrodynamics. II. Simulations, Astrophys. J. 858, 124 (2018).

[32] M. Dvornikov and V. B. Semikoz, Magnetic helicity evolution in a neutron star accounting for the Adler-Bell-Jackiw anomaly, J. Cosmol. Astropart. Phys. 08 (2018) 021. 